\title{
Corrosividad atmosférica del cobre y del acero en dos localidades de Villahermosa, Tabasco
}

\section{Corrosivity Index Copper and Steel at Two Locations in Villahermosa, Tabasco}

\author{
Tejero-Rivas María Candelaria \\ División Química \\ Universidad Tecnológica de Tabasco \\ Correo:tercy25@hotmail.com \\ Del Ángel-Meraz Ebelia \\ Universidad Juárez Autónoma de Tabasco, DAIA \\ Correo:ebelia.delangel@ujat.mx
}

\author{
Bautista-Margulis Raúl Germán \\ Ciencias Biológicas \\ Universidad Juárez Autónoma de Tabasco \\ Correo:margulisrg@hotmail.com
}

\author{
Hernández-Morales Nancy Elena \\ División Química \\ Universidad Tecnológica de Tabasco \\ Villahermosa, Tabasco México. \\ Correo:n_elena81@hotmail.com
}

Información del artículo: recibido: octubre de 2013, reevaluado: noviembre de 2013, aceptado: marzo de 2014

\section{Resumen}

Este trabajo presenta un estudio de la corrosión atmosférica del cobre y del acero al carbono realizado en dos ambientes de Villahermosa, Tabasco durante seis meses. El sitio de ensayo de la zona industrial se ubicó en el Instituto Tecnológico de Villahermosa (ITVH) y el sitio rural-urbano en la Universidad Tecnológica de Tabasco (UTTAB). Se utilizó el método alambre sobre tornillo de acuerdo a la Norma ASTM G116-93; el aluminio en combinación con un tornillo de acero al carbón proporcionó el índice de corrosividad marina (MA), el tornillo de cobre da el índice de corrosividad industrial (IA) y el tornillo de plástico nylon da el índice de corrosividad rural-urbano (RUA). La determinación de los contaminantes atmosféricos (cloruros y dióxido de azufre), se obtuvo con los métodos de candela húmeda y platos de sulfatación de acuerdo con la Norma ISO 9225. Se realizaron estudios de morfología en los productos de corrosión formados en las probetas de tornillo, aplicando microscopía electrónica de barrido acoplado con energías dispersivas. Los productos de corrosión que se formaron en la superficie del cobre y el acero al carbono, tienen una morfología en forma de ampolla, características de la adición de sales solubles, particularmente sulfatos y cloruros, identificados en las dos estaciones.

\section{Descriptores:}

- acero al carbono

- cobre

- contaminante

- corrosión 


\begin{abstract}
This paper presents a study of the atmospheric corrosion of copper and carbon steel made in two environments Villahermosa, Tabasco for six months. The test site of the industrial zone started Villahermosa Institute of Technology (ITVH) and rural-urban site at the Technological University of Tabasco (UTTAB). Aluminum in combination with a screw carbon steel provided the index marine corrosivity (MA), the brass screw gives the index of industrial corrosivity (IA; wire method of screw according to ASTM G116 -93 was used) and the plastic screw nylon gives the rate of rural-urban corrosivity (RUA). The determination of air pollutants (sulfur dioxide and chlorides), was with the methods of wet candle and sulfation plates according to ISO 9225. Morphology studies were performed on the corrosion products formed on the specimens screw, using scanning electron microscopy coupled with energy dispersive. The corrosion products that formed on the surface of copper and carbon steel, having a bulb-shaped morphology characteristic of the addition of soluble salts, particularly sulphates and chlorides, were identified in the two stations.
\end{abstract}
Keywords:
- carbon steel
- copper
- contaminant
- corrosion

\section{Introducción}

Actualmente uno de los problemas más graves causados por la contaminación ambiental, es la corrosión de los materiales. Las estructuras metálicas expuestas a la atmósfera como: monumentos, puentes, construcciones, etcétera, se deterioran debido a la corrosión atmosférica. El grado de deterioro de los materiales se relaciona directamente con el tipo de atmósfera al que se encuentra expuesto el metal, los factores climáticos y los contaminantes son los que determinan el tipo de productos de corrosión que se forman en la superficie de los metales (Del Ángel et al., 2009). La contaminación atmosférica se debe a la presencia en el aire de materias o formas de energía que impliquen riesgo, daño o molestia grave para las personas y bienes, pueden atacar a distintos materiales, reducir la visibilidad o producir olores desagradables (Villada et al., 2009).

Los principales mecanismo de contaminación atmosférica son los procesos industriales que implican combustión en las industrias, así como fuentes móviles y fuentes de área que generan dióxido de carbono y monóxido de carbono, óxidos de nitrógeno y azufre, entre otros contaminantes y las condiciones climáticas (Valdés et al., 2010). Estudios mundiales han demostrado que el costo total de las pérdidas de metales por corrosión es por lo menos 4-5\% del PIB de un país (Yuantai y Ying, 2010).

Muchos trabajos se han realizado hasta la fecha para determinar el comportamiento de diferentes materiales frente a la corrosión en distintos lugares y utilizando diferentes materiales y técnicas, como probetas planas y probetas de alambre sobre tornillo. Estudios realizados por el método de alambre sobre probeta en el estado de Veracruz reportaron un alto índice de co- rrosividad en zonas industriales (Ramírez et al., 2008). En Villahermosa se han realizado trabajos acerca de la corrosividad como los de Garcés (2002), en este estudio se evaluó el desempeño de materiales como el aluminio, cobre, latón y zinc, en donde se encontraron velocidades de corrosión alta $\left(\mathrm{C}_{5}\right)$. Del Ángel et al. (2009) realizaron estudios de corrosión atmosférica del acero galvanizado, registrando velocidades de corrosión media $\left(\mathrm{C}_{3}\right)$. En ambos estudios se calculó la velocidad de corrosión por el método de probetas planas. Este es el primer trabajo desarrollado en Tabasco sobre el índice de corrosividad atmosférica, utilizando el método de probeta de alambre sobre tornillo en dos zonas de estudio.

\section{Experimental}

Los sitios de ensayo fueron en el Instituto Tecnológico de Villahermosa Tabasco (ITVH) identificado como zona industrial y en la Universidad Tecnológica de Tabasco (UTTAB) como una zona rural-urbana. La exposición de las probetas se realizó de acuerdo a las especificaciones de la norma ISO 8565, el panel se orientó directamente de frente a los vientos dominantes en un lugar alto y sin obstáculos que obstruyan la incidencia directa de los vientos (figura 1).

Banco de pruebas de alambre sobre tornillo

Esta técnica está basada en la evaluación de la pérdida de masa que experimenta un alambre metálico enrollado en la rosca de un tornillo que suele tener un comportamiento catódico respecto al alambre (Linares, 2009). El alambre de aluminio se utiliza en combinación 
con un tornillo de acero al carbón que proporciona el índice de corrosividad marina (AM), el tornillo de cobre se utiliza para el índice de corrosividad industrial (AI) y el tornillo de plástico nylon se considera para el índice de corrosividad rural-urbano (RUA).

Para la preparación de las probetas de alambre sobre tornillo, se utilizaron tornillos de acero al carbón 1045, cobre y de plástico (nylon). Las dimensiones utilizadas en los tornillos fueron de $1.5 \mathrm{~cm}$ de diámetro y 12 $\mathrm{cm}$ de longitud. El alambre de aluminio comercial es de $1.5 \mathrm{~mm}$ de diámetro y $1 \mathrm{~m}$ de longitud. El alambre fue enrollado sobre la cuerda del tornillo y sujetado en sus extremos por dos orificios preparados en cada tornillo. Antes del montaje, los tornillos se limpiaron de acuerdo a la norma ISO 9226 y 8407.

Para la determinación del $\mathrm{SO}_{2}$ atmosférico, se empleó el método denominado "platos de sulfatación", estos se recolectaron después de un mes de exposición y se les realizó un análisis químico basado en la norma ISO 9225:1992. Para la determinación de cloruros atmosféricos, se empleó el método denominado "candela húmeda". Las muestras se analizaron cada mes en el laboratorio de acuerdo con la norma ISO 9225:1992. La limpieza de los tornillos de acero, cobre y alambre de aluminio se realizó utilizando la técnica gravimétrica (pérdida de masa); se eliminaron los productos de corrosión de acuerdo a la Norma ISO 8407. Para la estimación de los índices de corrosividad se aplicó la ecuación 1 en la forma descrita por la norma ASTM 116-93. Los resultados obtenidos, permitieron caracterizar el índice de corrosividad de cada material en función de la velocidad de corrosión para un periodo menor de un año de acuerdo con la norma ISO 9223 (Genesca, 1994).

$\mathrm{ICA}=(\Delta P / P \mathrm{i}) * 100$

Donde $\Delta P$ es la pérdida de peso del aluminio, $P$ i es el peso inicial que tenía el alambre antes de ser expuesto a la atmosfera.

\section{Parámetros climáticos}

En un clima tropical-húmedo, las fuertes variaciones de los parámetros climáticos, como la humedad relativa, la temperatura, la velocidad del viento, las precipitaciones pluviales, la radiación solar, entre otros, afectan de una manera relevante muchos procesos físicos, químicos y biológicos (Del Án- gel et al., 2007). Para la caracterización ambiental de los sitios de ensayo se recurrió a datos meteorológicos. Para el análisis de los parámetros climáticos se procesaron los valores horario de temperatura $\left(\mathrm{T}^{\circ} \mathrm{C}\right)$, humedad relativa (HR) del aire y velocidad de viento promedio por mes en ambos sitios de ensayo, registrados y proporcionados por las estaciones meteorológicas locales de la Comisión Nacional del Agua (CONAGUA).

\section{Morfología del producto de corrosión}

Para determinar la morfología de los productos de corrosión se utilizó el microscopio electrónico de barrido (MEB), con un detector EDS (espectroscopia de energía dispersiva) que permitió obtener información diversa como morfología y composición elemental.

\section{Resultados y discusión}

Durante el periodo de estudio se realizó un registro mensual de la velocidad de deposición de dióxido de azufre $\left(\mathrm{SO}_{2}\right)$ y NaCl , como principales agentes corrosivos para los metales. $\mathrm{El} \mathrm{SO}_{2}$ es uno de los agentes más importante para la corrosión de materiales, ya que participa activamente en la interface metal-ambiente de película húmeda formada sobre la superficie del material y de esta manera puede acelerar su corrosión atmosférica. Las concentraciones mensuales de deposición de $\mathrm{SO}_{2}$ variaron durante los meses de estudio y se observó un aumento en sus valores durante los eventos de quema de pastizales en zonas aledañas del ambiente rural-urbano de la UTTAB, donde fue más acentuada en los meses de mayo y junio. En el ambiente del ITVH se registró un aumento en la deposición de este contaminante agresivo en la mayoría de los meses, debido a su cercanía a ciertas industrias y al tráfico vehicular. La agresividad corrosiva del ambiente de ITVH es muy baja $\left(P_{0}\right)$, según la norma ISO 9223:92, debido a que los
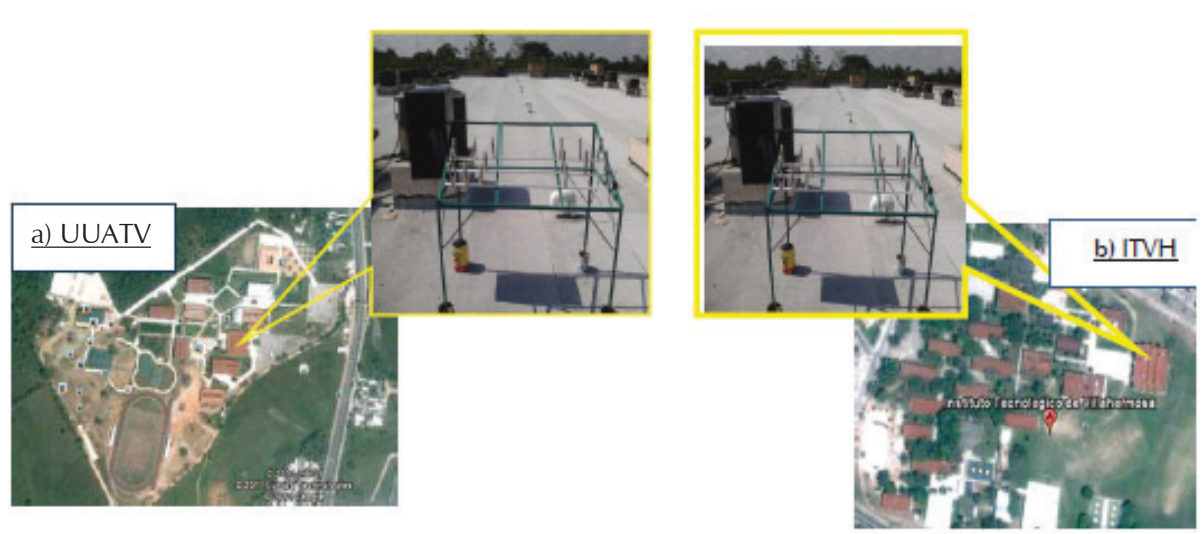

Figura 1. Panel de exposición de las muestras de acero y cobre, inclinado $45^{\circ}$ 
valores de deposición diaria de este contaminante estuvieron en el intervalo de 0.06 a $8.09 \pm 3.74 \mathrm{mg} / \mathrm{m}^{2}$.d (tabla 1), mientras en el ambiente rural-urbano de la UTTAB los valores respectivos fueron de 0.05 a $9.08 \pm$ $3.61 \mathrm{mg} / \mathrm{m}^{2} \mathrm{~d}$ con una categoría de agresividad muy baja $\left(P_{0}\right)$.

Investigaciones realizadas en ciudades con climas tropicales-húmedos reflejan mayor agresividad hacia los metales, principalmente por el efecto de contaminantes de iones cloruros y dióxido de azufre, el primero se incorpora a la atmósfera desde el mar. La cantidad de cloruros depositada en los sitos de ensayo indica que este contaminante muestra una relación directa con la distancia de la costa, asimismo su presencia depende de los períodos con frentes fríos y velocidad de viento. Debido a que el ambiente de ITVH se encuentra a $76 \mathrm{~km}$ de la costa del Golfo de México, la cantidad de cloruros depositados sobre la superficie del metal en el periodo de este estudio $\left(0.17-1.39 \pm 0.43 \mathrm{mg} / \mathrm{m}^{2} \mathrm{~d}\right)$ fue en general mayor, comparada con la cantidad depositada en el ambiente urbano de Villahermosa $(0.06-0.97 \pm 0.38$ $\mathrm{mg} / \mathrm{m}^{2}$ ) (tabla 2). De esta manera, según la clasificación propuesta por la norma ISO 9223:92, la agresividad corrosiva para los dos ambientes fue muy baja $\left(S_{O}\right)$.

Tabla 1. Velocidad mensual de deposición $\left(\mathrm{V}_{\text {dep }}\right)$ del $\mathrm{SO}_{2}\left(\mathrm{mg} / \mathrm{m}^{2}\right.$.d) según la norma ISO 9223:1992, para los dos sitios de ensayo

\begin{tabular}{ccccc}
\hline $\begin{array}{c}\text { Tiempo } \\
\text { (Ene-Jun 2012) }\end{array}$ & $\begin{array}{c}V_{\text {dep }} \mathrm{SO}_{2} \\
\left(\mathrm{mg} / \mathrm{m}^{2} . \mathrm{d}\right) \\
\mathrm{UTTAB}\end{array}$ & $\begin{array}{c}\text { Categoría } \\
\text { ISO 9223:1992 }\end{array}$ & $\begin{array}{c}V_{\text {dep }} \mathrm{SO}_{2} \\
\left(\mathrm{mg} / \mathrm{m}^{2} . \mathrm{d}\right) \\
\mathrm{ITVH}\end{array}$ & $\begin{array}{c}\text { Categoría } \\
\text { ISO 9223:1992 }\end{array}$ \\
\hline Enero & 0.10 & $\mathrm{P}_{\mathrm{o}}$ & 0.06 & $\mathrm{P}_{\mathrm{o}}$ \\
Febrero & 0.05 & $\mathrm{P}_{\mathrm{o}}$ & 0.30 & $\mathrm{P}_{\mathrm{o}}$ \\
Marzo & 1.83 & $\mathrm{P}_{\mathrm{o}}$ & 0.47 & $\mathrm{P}_{\mathrm{o}}$ \\
Abril & 4.35 & $\mathrm{P}_{\mathrm{o}}$ & 2.98 & $\mathrm{P}_{\mathrm{o}}$ \\
Mayo & 9.08 & $\mathrm{P}_{\mathrm{o}}$ & 8.09 & $\mathrm{P}_{\mathrm{o}}$ \\
Junio & 6.18 & $\mathrm{P}_{\mathrm{o}}$ & 7.71 & $\mathrm{P}_{\mathrm{o}}$ \\
\hline
\end{tabular}

Tabla 2. Velocidad mensual de deposición $\left(V_{\text {dep }}\right)$ del $\mathrm{NaCl}\left(\mathrm{mg} / \mathrm{m}^{2} . \mathrm{d}\right)$ en los ambientes de estudio

\begin{tabular}{ccccc}
\hline $\begin{array}{c}\text { Tiempo } \\
\text { Ene-Jun 2012) }\end{array}$ & $\begin{array}{c}V_{\text {dep }} \mathrm{NaCl} \\
\left(\mathrm{mg} / \mathrm{m}^{2} . \mathrm{d}\right)\end{array}$ & $\begin{array}{c}\text { Categoría } \\
\mathrm{UTTAB}\end{array}$ & $\begin{array}{c}V_{\text {dep }} \mathrm{NaCl} \\
\left(\mathrm{mg} / \mathrm{m}^{2} . \mathrm{d}\right) \\
\mathrm{ISO} 9223: 1992\end{array}$ & $\begin{array}{c}\text { Categoría } \\
\text { ISO 9223:1992 }\end{array}$ \\
\hline Enero & 0.14 & $\mathrm{~S}_{\mathrm{o}}$ & 0.64 & $\mathrm{~S}_{\mathrm{o}}$ \\
Febrero & 0.06 & $\mathrm{~S}_{\mathrm{o}}$ & 0.17 & $\mathrm{~S}_{\mathrm{o}}$ \\
Marzo & 0.68 & $\mathrm{~S}_{\mathrm{o}}$ & 0.82 & $\mathrm{~S}_{\mathrm{o}}$ \\
Abril & 0.97 & $\mathrm{~S}_{\mathrm{o}}$ & 1.39 & $\mathrm{~S}_{\mathrm{o}}$ \\
Mayo & 0.74 & $\mathrm{~S}_{\mathrm{o}}$ & 0.92 & $\mathrm{~S}_{\mathrm{o}}$ \\
Junio & 0.16 & $\mathrm{~S}_{\mathrm{o}}$ & 0.38 & $\mathrm{~S}_{\mathrm{o}}$ \\
\hline
\end{tabular}

Tabla 3. Valores mensuales de datos meteorológicos de los sitios de ensayo

\begin{tabular}{ccccccc}
\hline \multirow{2}{*}{$\begin{array}{c}\text { Tiempo } \\
\text { (Dic 2011-Jun 2012) }\end{array}$} & UTTAB & ITVH & UTTAB & ITVH & UTTAB & ITVH \\
\hline Diciembre & 3.06 & 3.24 & 89.45 & 91.75 & 30.14 & 24.11 \\
Enero & 3.97 & 3.24 & 85.44 & 89.37 & 27.85 & 23.59 \\
Febrero & 4.26 & 3.6 & 98.75 & 89.9 & 26.37 & 24.07 \\
Marzo & 5.15 & 4.11 & 75.86 & 78.22 & 27.33 & 26.04 \\
Abril & 3.53 & 3.71 & 75.41 & 76.45 & 30.04 & 26.92 \\
Mayo & 3.78 & 3.1 & 79.99 & 83.39 & 30.15 & 27.85 \\
Junio & 4.04 & 3.9 & 79.05 & 83.05 & 30.16 & 28.28 \\
\hline
\end{tabular}


En la tabla 3 se muestran los valores de temperatura, humedad relativa y velocidad de vientos, tomados con base a seis meses durante el periodo de exposición de las probetas en el ITVH y UTTAB. En las zonas tropicales, donde las variaciones de la temperatura durante el día son generalmente mayores, la humedad relativa cambia considerablemente en el curso del día. Se sabe que durante la quema de biomasa, se liberan importantes cantidades de dióxido de azufre (Graf et al., 1997; Figueruelo y Dávila, 2004). En la ciudad de Villahermosa, esta actividad se realiza de manera programada durante la época de estiaje entre los meses de abril y junio, alcanzando su pico máximo en marzo, mismos que se pueden relacionar con las velocidades de viento altas reportadas entre los meses de febrero y marzo. Las concentraciones de cloruros y $\mathrm{SO}_{2}$ observados tuvieron un impacto notorio en el comportamiento corrosivo de la atmósfera en las estaciones con fuertes vientos y temperaturas secas.

\section{Índices de corrosividad atmosférica}

El monitoreo de la corrosividad atmosférica del ITVH (tabla 5), indica que las condiciones meteorológicas influyen en las condiciones agresivas de la atmósfera. En cuanto a los índices de corrosividad evaluados se puede decir que los de la atmósfera marina (AM) se incrementan considerablemente en el segundo y tercer bimestre, lo cual se puede atribuir a la temporada de nortes, que arrastra gran cantidad de cloruros desde las costas del Golfo de México, como se observa en la velocidad de viento reportada durante los meses de marzo- abril en los que alcanzaron las cantidades más altas de 3.71 y 4.11 $\mathrm{m} / \mathrm{s}$. Sin embargo, la atmósfera industrial (AI), también se incrementa excesivamente; no obstante, el contenido de azufre se considera bajo, lo cual se atribuye también a la presencia de los cloruros que resultaron altos en los meses de abril y mayo. Esto resultó por la alta actividad urbana propia del lugar, al grado que rebasaron los límites establecidos para una atmósfera industrial. Los índices para una atmósfera rural y urbana (RUA), registraron un incremento considerable en el tercer bimestre principalmente, rebasando también los límites permitidos, lo que seguramente se debe a las condiciones climáticas, ya que por su intensidad combinan las condiciones de humedad y temperatura que arrastran los vientos en la temporada de nortes principalmente (tabla 4).

En la zona de estudio de la Universidad Tecnológica de Tabasco, se muestran índices de corrosividad en aumento en el ambiente marino (AM), pero que se encuentran dentro del límite estándar. La atmósfera industrial (AI), presenta durante el primer bimestre corrosividad baja, la cual aumenta en el primero y segundo bimestre, propio de las actividades urbanas realizadas. A estas condiciones atmosféricas les añadimos el efecto de los parámetros contaminantes como los cloruros, que no están tan altos como en la costa; sin embargo son niveles suficientes para incrementar la corrosividad. Los índices para una atmósfera rural y urbana registraron una corrosividad relativamente baja en los dos primeros bimestres, rebasando también el límite permitido en el tercero, lo cual significa que en este bimestre se alcanzó una zona de tipo urbano-industrial (tabla 6).

El estudio de morfología se realizó en las muestras de acero al carbono y cobre, expuestas a la atmósfera de los dos sitios de ensayo obteniéndose un espectrograma por cada material, a partir de ellos se realizó la identificación de los productos de corrosión presentes en la muestra. En el siguiente apartado se representan las micrografías y el análisis EDS de los productos de corrosión que fueron identificados en cada material por cada uno de los sitios de ensayo e incluyen la muestra testigo (mes cero).

En la figura 3 se muestra la caracterización morfológica superficial de la muestra testigo de acero al carbono instalado en ITVH, con la técnica de microscopia de barrido electrónico, se observa claramente la aleación de materiales como el hierro en el acero al carbono, por lo que se puede confirmar que el material estaba puro, libre de elementos como azufre y cloro, contaminantes presentes en la atmósferas y causantes de los productos de corrosión.

El análisis superficial de las muestras de acero al carbono instaladas en el ITVH utilizando el microscopio electrónico de barrido (MEB) en enero del 2012, muestran porosidades en la superficie y formación de ampollas debido a la presencia de productos de corrosión (figura 4). De igual manera en la figura $4 \mathrm{~b}$, se muestra el análisis elemental (EDS), en donde se observa la aparición de las primeras fases de productos de corrosión. Se detectó la presencia de Azufre y Cl, la fase de Fe continúa siendo la mayoritaria en la muestra de acero al Carbono, de igual manera elementos como el Oxígeno y Silicio, este último como parte de $1 \%$ de su composición química.

En cuanto a lo que corresponde a la caracterización morfológica superficial de la muestra testigo de acero al carbono instalado en UTTAB, la figura 5, muestra al Hierro, silicio, manganeso y carbono como parte de la aleación de este material.

El espectrograma del primer mes de exposición del acero al carbono en el ambiente de la UTTAB (figura 6), presenta elementos como $\mathrm{Ca}$, $\mathrm{Fe}$ y $\mathrm{O}$, que son los últimos productos mayoritarios. La presencia de $\mathrm{Cl}$ y $\mathrm{S}$ en la 
Tabla 4.- Clasificación de la corrosividad basado en la norma ISO 9223

\begin{tabular}{cccc}
\hline Simbología & Corrosividad & Atmósfera & Estándar \\
\hline & Baja & Costera & $0.4-9$ \\
Aceptable & Industrial & $0.15-1.8$ \\
& Agresiva & Rural & 1 a 2 \\
& Muy agresiva & & \\
\hline
\end{tabular}

Tabla 5. Índice de corrosividad en el ITVH

\begin{tabular}{cccc}
\hline Tiempo & AM, \% & AI, \% & RUA\% \\
\hline Bimestre 1 & 5.5 & 0.7 & 0.5 \\
Bimestre 2 & 15 & 1.7 & 1.1 \\
Bimestre 3 & 13.9 & 6.5 & 3.6 \\
\hline
\end{tabular}

Figura 2. Mapa de ubicación de los sitios de ensayo

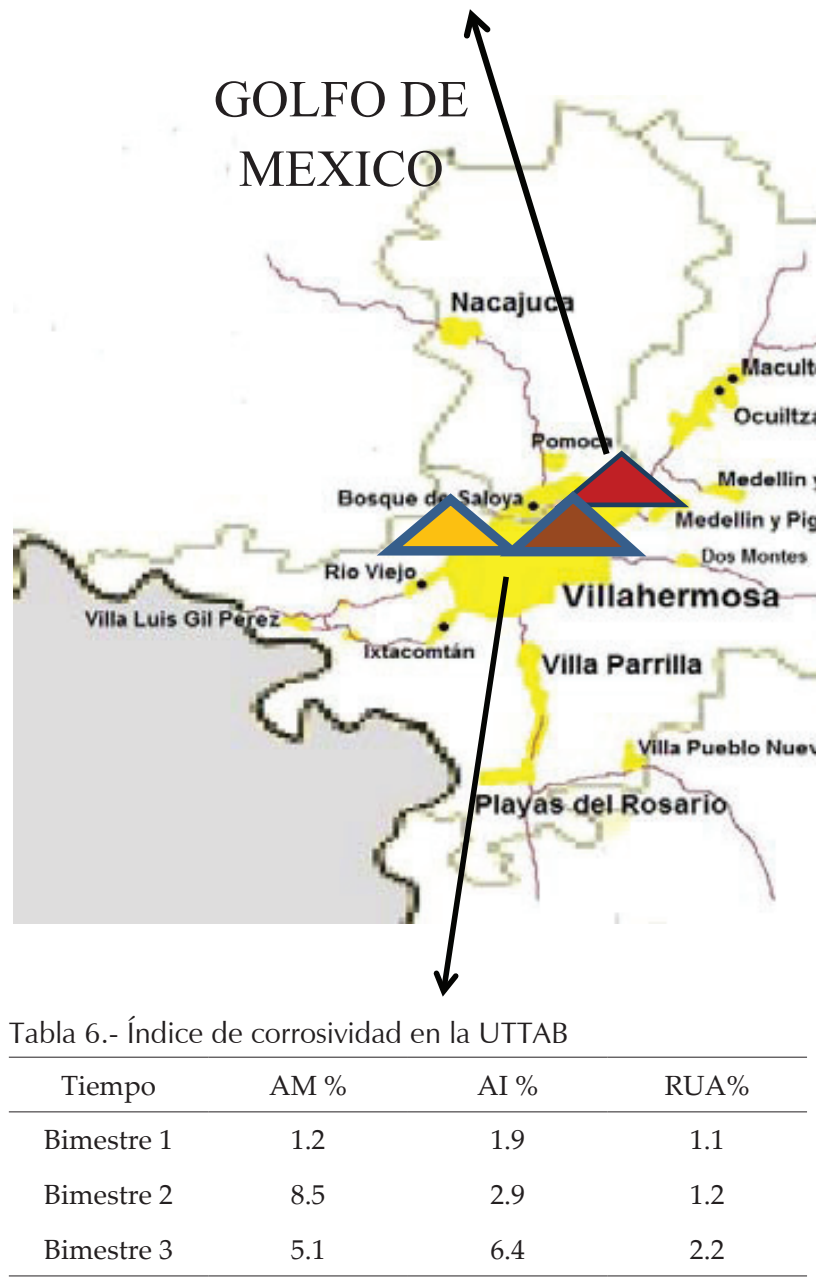

muestra indica que, aunque la velocidad de deposición de estos contaminantes provenientes del ambiente de la UTTAB fueron relativamente bajos, estos influyen directamente en la velocidad de corrosión de este material.
En la figura 7 se muestra la caracterización morfológica superficial de la muestra testigo del cobre instalado en ITVH, también el material en estado libre de elementos contaminantes presentes en las atmósferas y causantes de los productos de corrosión.

A través del microscopio electrónico de barrido se observó corrosión por picaduras en la probeta de cobre, que el ojo humano no puede observar a simple vista debido a que las hendiduras son muy pequeñas. El espectrograma muestra presencia de metales como calcio (Ca), silicio ( $\mathrm{Si}$ ), aluminio $(\mathrm{Al})$ y elementos no metálicos contaminantes con el azufre (S) y iones cloruros $\left(\mathrm{Cl}^{-}\right)$. Se sugiere que la presencia de aluminio forma una placa de óxido conocido como alúmina sobre la superficie, formación característica de este metal con el oxígeno atmosférico (figura 8).

El estudio de MEB realizado al tornillo de cobre testigo en la UTTAB muestra el material limpio, sin contaminantes o incrustaciones (figura 9).

La característica que hace más interesante el análisis de la corrosión atmosférica del cobre es que sus productos de corrosión, además de ser insolubles en el agua de lluvia, son compuestos que incluyen a los contaminantes. Por esta razón se utilizaron, para censar el nivel de contaminación de un determinado sitio y para establecer el origen de elementos espúreos no investigados a través de los procedimientos especificados en las respectivas normas de evaluación de las categorías de corrosividad.

La muestra de cobre expuesta durante el primer mes, presenta metales como Fierro (Fe), Aluminio (Al) y Silicio ( $\mathrm{Si}$ ) como se aprecia en la figura 10. Al mismo tiempo que se observan elementos como Oxígeno $(\mathrm{O})$, Cloro (Cl-) y Azufre (S).

\section{Conclusiones}

En la zona de estudio ITVH se manifestó un índice de corrosividad agresivo para los materiales estudiados de 
a)

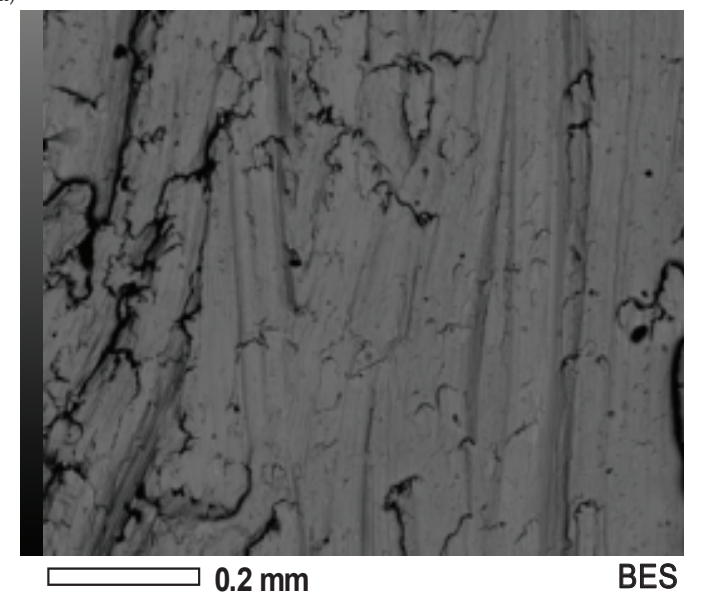

b)

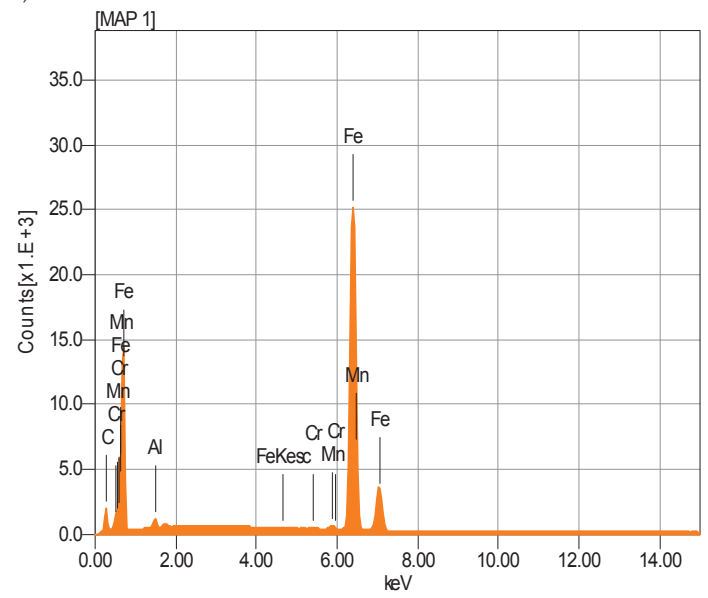

Figura 3. Micrografía y análisis EDS de la muestra testigo del acero al carbono del ITVH

a)

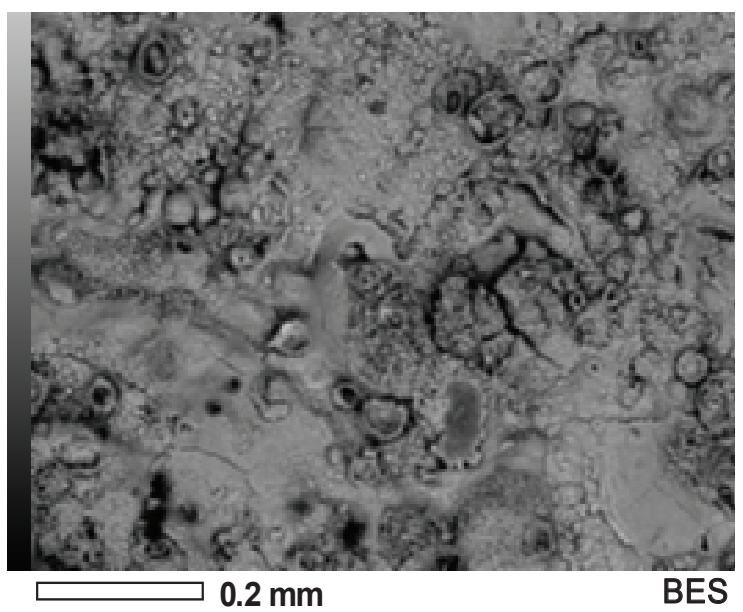

b)

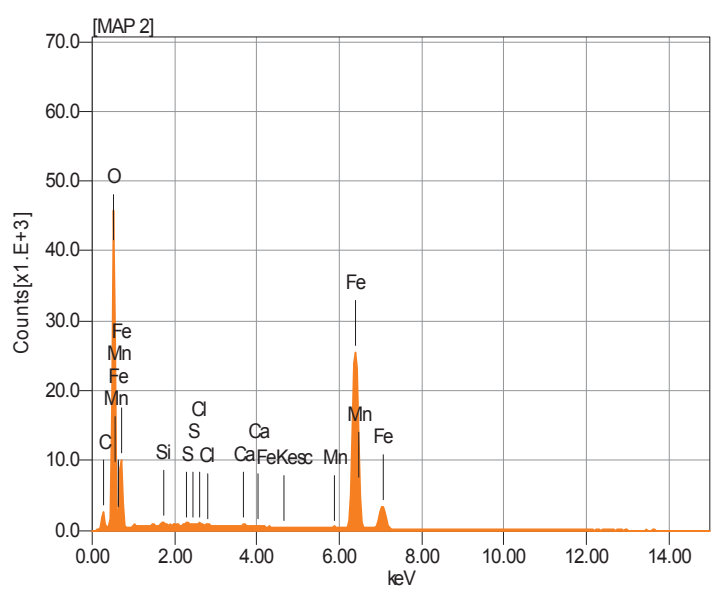

Figura 4. Micrografía y análisis EDS del acero correspondiente al primer mes de exposición en el ITVH (enero 2012)

a)

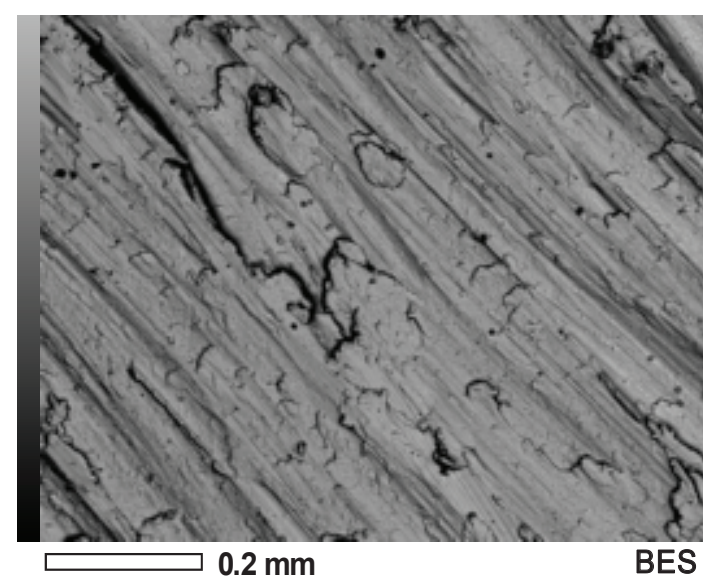

b)

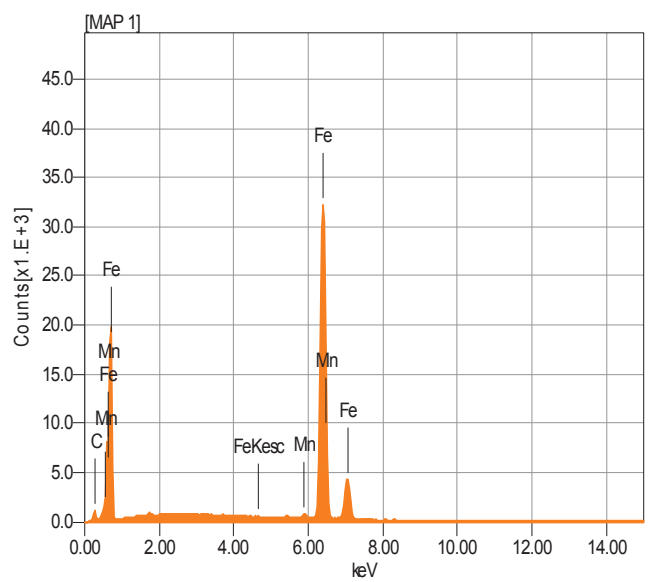

Figura 5. Micrografía y análisis EDS de la muestra testigo del acero al carbono en la UTTAB 


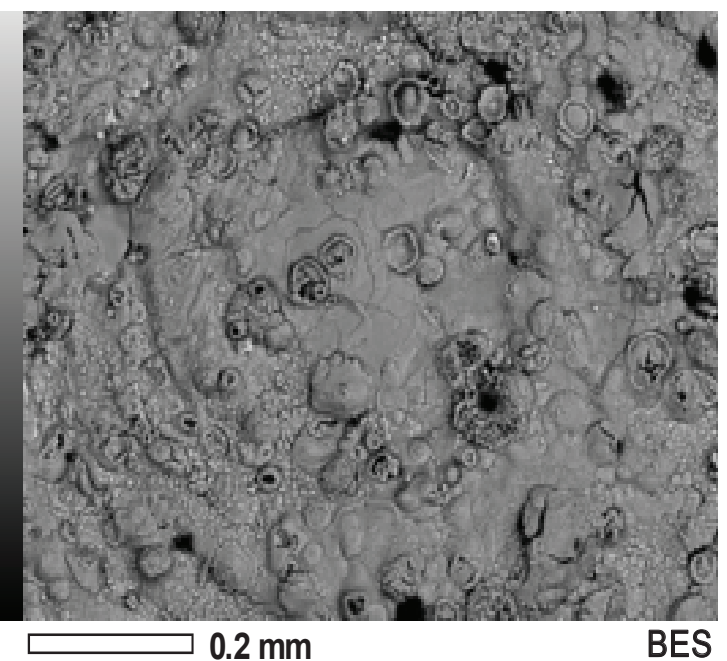

b)

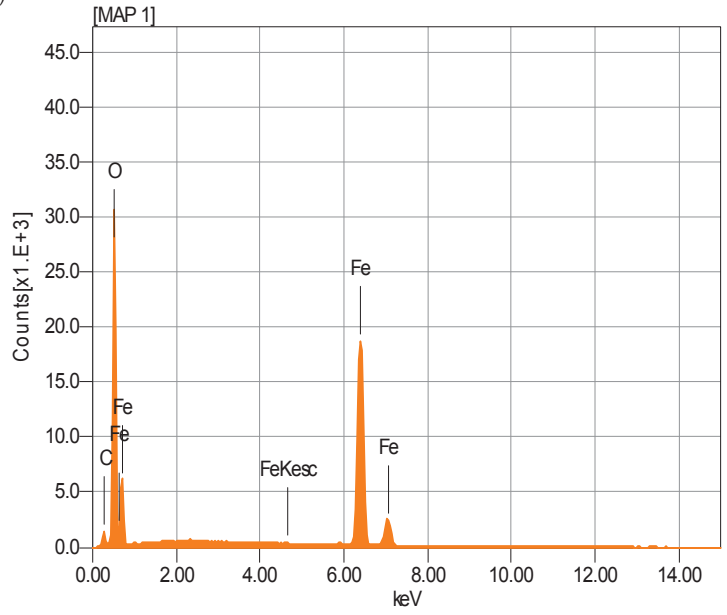

Figura 6. Micrografía y análisis EDS del acero correspondiente al primer mes de exposición en la UTTAB (enero 2012)
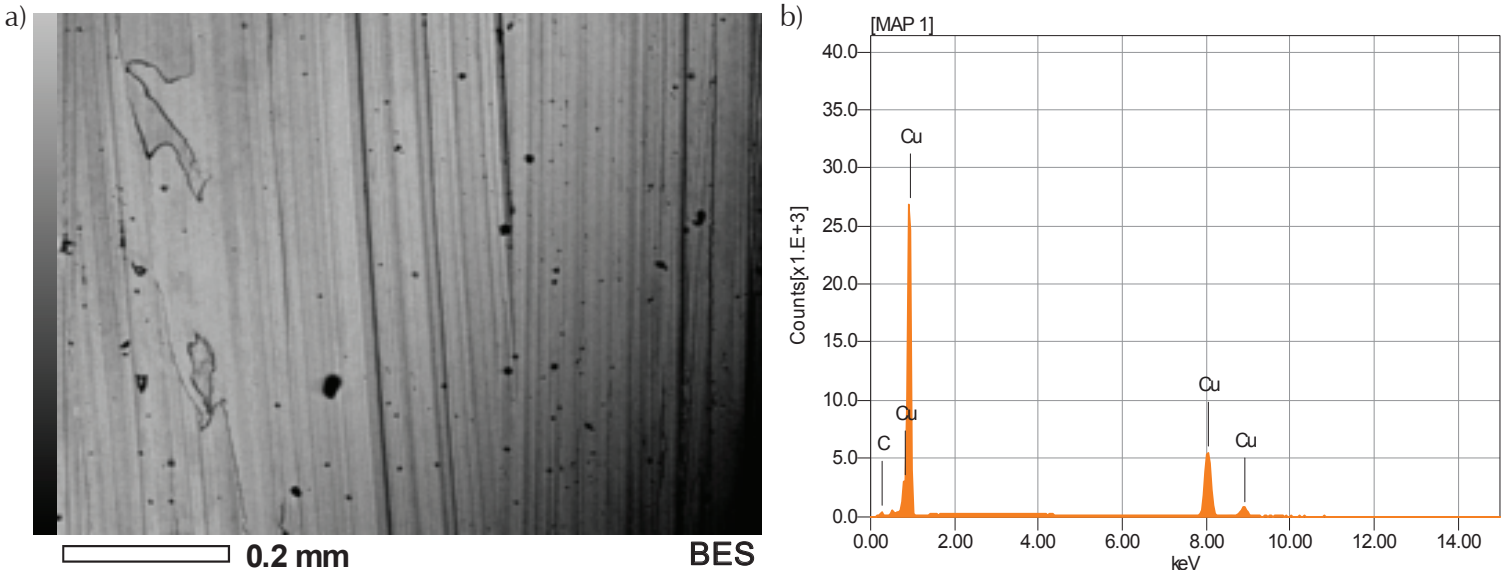

Figura 7. Micrografía y análisis EDS de la muestra testigo del cobre en el ITVH
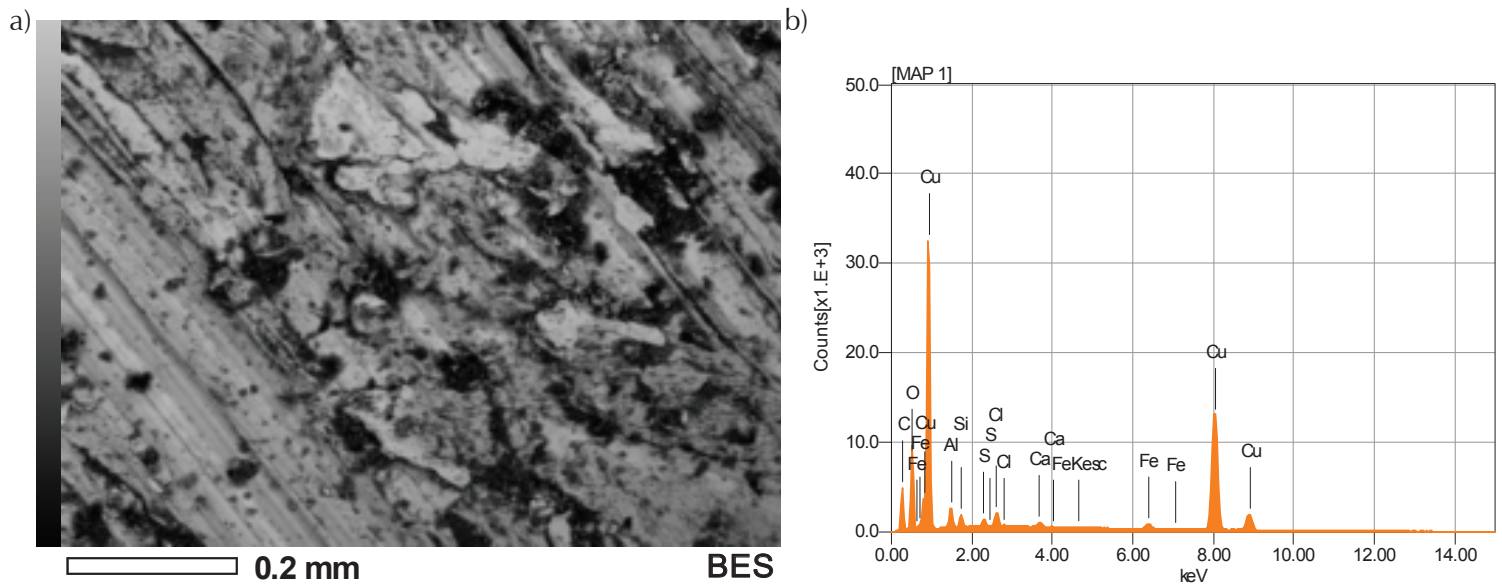

Figura 8. Micrografía y análisis EDS del cobre correspondiente al primer mes de exposición en el ITVH (enero 2012) 
a)

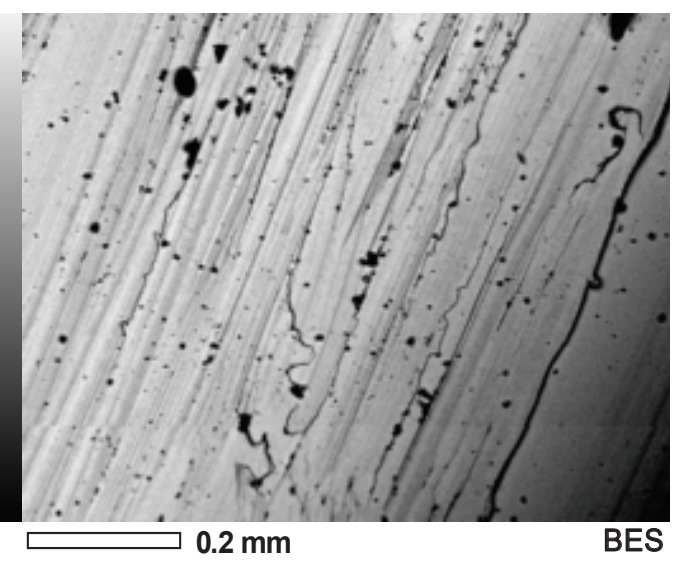

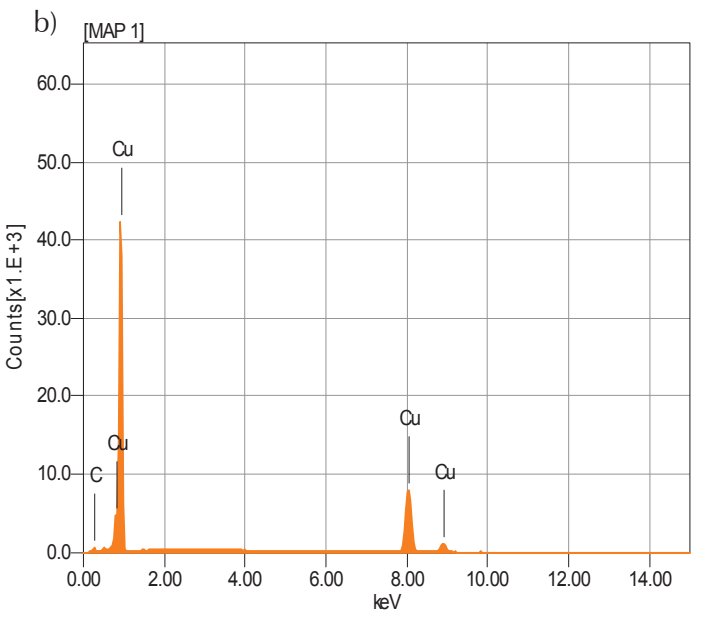

Figura 9. Micrografía y análisis EDS del cobre correspondiente al primer mes de exposición en el UTTAB (enero 2012)

a)

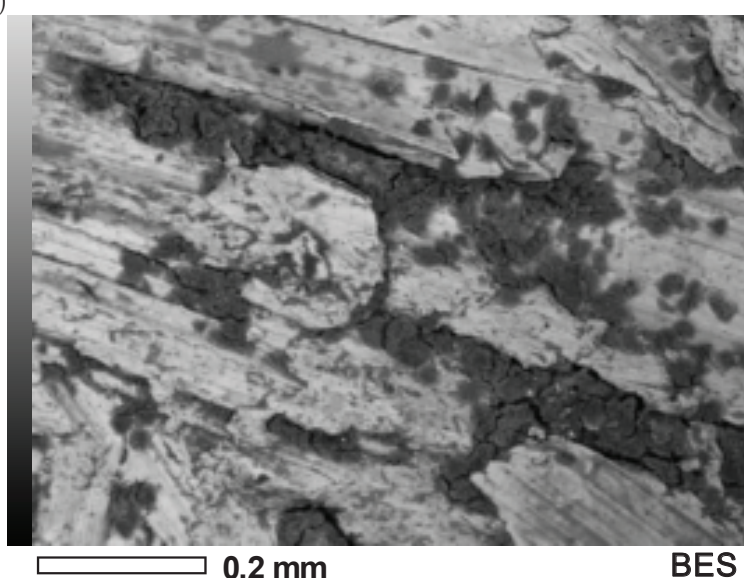

b)

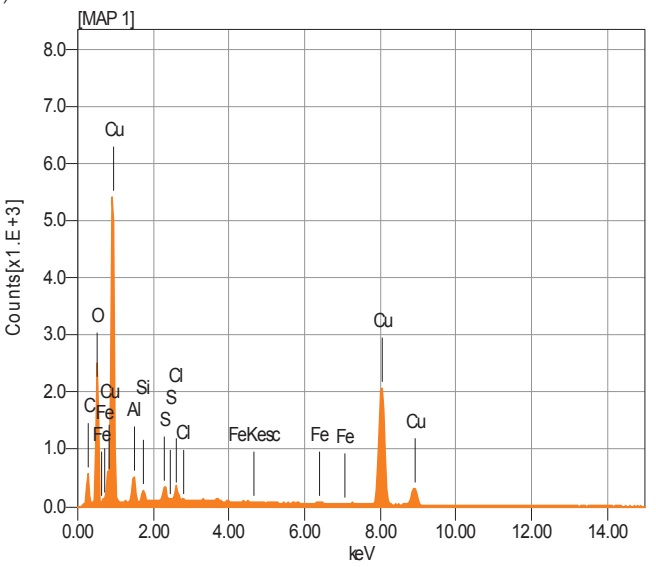

Figura 10. Micrografía y análisis EDS del cobre correspondiente al primer mes de exposición en el UTTAB (enero 2012)

la atmósfera marina (AM), el índice de corrosividad fue aceptable durante el primero y segundo bimestre, volviéndose muy agresivo en el tercer bimestre. En el caso de la atmósfera industrial (AI), se mostró un índice de agresividad muy alto en los tres bimestres de estudio. Los índices para una atmósfera rural y urbana (RUA), registraron un incremento de corrosividad aceptable en los dos primeros bimestres, rebasando también los límites permitidos en el tercero. En la zona de estudio de la UTTAB, se muestran incrementos en los índices de corrosividad en cada bimestre en el ambiente (AM); mientras que la atmósfera industrial (AI) presenta en el primer bimestre corrosividad baja, que aumenta en el primero y segundo bimestres, propio de las actividades urbanas realizadas. Los índices para una atmósfera rural y urbana registraron una corrosividad relativamente baja en los dos primeros bimestres, y muy agresiva en el tercero, no obstante que el contenido de azufre fue bajo en ambos sitios de ensayo. Las categorías de agresividad corrosiva con base en los contaminantes $\left(\mathrm{SO}_{2} \mathrm{y}\right.$ $\mathrm{NaCl}$ ), con referencia en la norma ISO 9223:92, se encontró baja tanto para el $\mathrm{SO}_{2}\left(P_{0}\right)$ y los cloruros $\left(\mathrm{S}_{0}\right)$ en los dos sitios de ensayo.

La morfología de los productos de corrosión que se formaron en la superficie del acero presentó forma de ampolla, característica de la adición de sales solubles, particularmente sulfatos y cloruros, identificados en ambas estaciones. La interacción del haz de electrones de este equipo con el sólido origina electrones secundarios, Auger, electrones dispersados y Rayos X, que permiten identificar la posible formación de $\mathrm{SiO}_{2}, \mathrm{SO}_{3}$ $\mathrm{CaO}, \mathrm{MnO}$ y FeO en la superficie, esto sugiere que existe una penetración del oxígeno a la superficie del metal. El estudio de morfología en la placa de Cobre, muestra 
que los productos de corrosión que se formaron en la superficie se debe a la presencia de oxígeno del ambiente, así como a la presencia de metales como Fierro $(\mathrm{Fe})$, Aluminio (Al) y Silicio (Si). Al mismo tiempo que se observan elementos $\mathrm{Cloro}\left(\mathrm{Cl}^{-}\right)$y Azufre (S), considerados fuertes agentes contaminantes.

\section{Referencias}

Del Ángel E., Veleva L., Acosta A.M. Agresividad atmosférica basada en el tiempo de humectación del clima tropical húmedo del estado de Tabasco. Universidad y Ciencia, volumen 25 (número 2), 2009: 111-120.

Del Ángel E., Veleva L., Acosta A.M. Etapas iniciales del zinc runoff en clima tropical Húmedo. Revista de Metalurgia, volumen 43 (número 2) marzo-abril 2007: 85-100

Figueruelo J. y Dávila M. Química física del ambiente y de los procesos medioambientales, editorial Reverté, España, 2004, pp. 591.

Garcés R. Evaluación de la velocidad de corrosión atmosférica del aluminio, cobre latón y zinc, tesis (licenciatura en química), Villahermosa Tabasco, Cunduacán, noviembre 2002, 84 p.

Genescá-Llongueras J. Más allá de la herrumbre III. Corrosión y medio ambiente, Paciencia, México, 1994.

Graf H., Feichter J., Langmann B. Volcanic sulphur emissions: Estimates of source strength and its contribution to the global sulfate distribution. Journal of Geophysical Research, volumen 102, 1997: 10727-10738.

ISO 8565. Corrosion of metal and alloys - atmospheric corrosion testing-general.

ISO 9225:1992. Corrosion of metal and alloys, classification of atmospheres. Measurement of pollution.

ISO 8407:91. Corrosion of metals and alloys removal of corrosion products from corrosion test specimens. International Organization for Standardization, Genova, 1991.
ISO 9223:1992. Corrosion of metals and alloys classification of atmospheres. International Organization for standardization, Genova, 1992.

Linares L., Bermúdez O., Taborda N. Estaciones aeroquímicas y meteorológicas para la construcción de mapas de corrosividad atmosférica en el sistema de potencia. Comité Nacional Venezolano, en: II Congreso Venezolano de Redes y Energia Electrica, noviembre de 2009.

Ma Y., Li Y., Wang F. The atmospheric corrosion kinetics of low carbon steel in a tropical marine environment. Corrosion Science, volumen 52 (número 5), 2010: 1796-1800.

Ramírez J., Hernández E., Pereyra D., Morales R. Índices de corrosividad en la región de Xalapa, Veracruz, en: XVII Congreso de la IMRC y VII Congreso NACE International Sección México en Cancún Q. Roo, agosto, 2008.

Valdés C., Corvo F., Pérez J., Portilla C., Fernández D. Contaminación ambiental y su influencia en las edificaciones, estudios preliminares. Revista CENIC, Ciencias Químicas, 2010.

Villada F., Molina J., Velilla E. Modelo de vida útil para estructuras acero-zinc utilizadas en la transmisión de energía eléctrica en Colombia. Inf. tecnol., volumen 20 (número 6), 2009: 57- 66.

\section{Este artículo se cita:}

\section{Citación estilo Chicago}

Tejero-Rivas, María Candelaria, Ebelia Del Ángel-Meraz, Raúl Germán Bautista-Margulis, Nancy Elena Hernández-Morales. Corrosividad atmosférica del cobre y del acero en dos localidades de Villahermosa, Tabasco. Ingeniería Investigación y Tecnología, XVI, 02 (2015): 197-206.

\section{Citación estilo ISO 690}

Tejero-Rivas M.C., Del Ángel-Meraz E., Bautista-Margulis R.G., Hernández-Morales N.E. Corrosividad atmosférica del cobre y del acero en dos localidades de Villahermosa, Tabasco. Ingeniería Investigación y Tecnología, volumen XVI (número 2), abril-junio 2015: 197-206.

\section{Semblanzas de los autores}

María Candelaria Tejero-Rivas. Es ingeniería bioquímica egresada del Instituto Tecnológico de Villahermosa, actualmente es docente en el Instituto Tecnológico de Villahermosa (ITVH) y la Universidad Tecnológica de Tabasco (UTTAB).

Ebelia del Angel-Meraz. Es ingeniera química egresada del Instituto Tecnológico de Cd, Madero, maestría en tecnología del petróleo y petroquímica (ITCM). Doctora en Corrosión, egresada del Programa de Corrosión del Golfo de México, trabaja como profesor investigador en la Universidad Juárez Autónoma de Tabasco. Sus áreas de investigación: corrosión atmosférica y desarrollo de materiales para control ambiental.

Raúl Germán Bautista-Margulis. Es ingeniero químico egresado de la Universidad Veracruzana, con maestría y doctorado por la Universidad de Sheffield en Inglaterra. Trabajó como investigador en el Instituto de Investigaciones Eléctricas y en el Centro de Investigación en Materiales Avanzados. Actualmente es profesor-investigador de la Universidad Juárez Autónoma de Tabasco y sus áreas de interés en la investigación son: contaminación atmosférica, sistemas de combustión y deterioro de materiales.

Nancy Elena Hernández-Morales. Es ingeniería química, egresada del Instituto Tecnológico de Villahermosa, actualmente es docente del Instituto Tecnológico de Villahermosa (ITVH) y la Universidad Tecnológica de Tabasco (UTTAB). 